\title{
Comunicação
}

[Comunication]

\section{Desempenho do polvo Octopus vulgaris alimentado com monodieta de mexilhão (Perna perna )}

[Performance of the Octopus vulgaris octopus fed on mussel (Perna perna) as monodiet]

\author{
P. Bastos ${ }^{1}$, I.M.M. dos Reis ${ }^{1}$, R.L. Costa ${ }^{2}$, J.F. Ferreira ${ }^{2}$
}

${ }^{1}$ Aluno de pós-graduação - Universidade Federal de Santa Catarina - Florianópolis, SC

${ }^{2}$ Universidade Federal de Santa Catarina - Florianópolis, SC

O polvo Octopus cf. vulgaris (Cuvier 1797) é espécie cosmopolita e tem grande potencial para a diversificação e inovação na aquicultura marinha devido ao rápido crescimento, à eficiente taxa de conversão alimentar e ao valor de mercado atrativo (Vaz-Pirez et al., 2004). É excelente fonte de proteína, pois possui de 75 a $85 \%$ de proteína bruta (peso seco) e tem alto índice de aproveitamento, isto é, $80-85 \%$ do total corporal pode ser aproveitado para consumo humano (Lee, 1994).

Apesar da potencialidade do O. vulgaris para maricultura, o cultivo dessa espécie em escala comercial ainda não é viável devido, principalmente, às altas mortalidades na fase de larvicultura (Iglesias e Fuentes, 2014). A produção industrial dessa espécie é realizada na Espanha, baseada na engorda de juvenis e subadultos capturados no ambiente. Na engorda comercial do $O$. vulgaris, é possível obter taxas de crescimento de $1,0 \mathrm{~kg} / \mathrm{mês}$ com dieta natural baseada em espécies de baixo valor comercial (bycatch) (Vaz-Pirez et al., 2004). Contudo, a variabilidade de espécies e a disponibilidade do bycatch como matéria-prima podem comprometer a sustentabilidade da engorda em longo prazo (Estefanell et al., 2013).

Até o momento, não há dieta artificial balanceada aplicável comercialmente para o cultivo do O. vulgaris. Embora existam avanços recentes com dietas inertes, essas dietas formuladas não promovem desempenho tão eficiente quanto as dietas naturais, baseadas em espécies de baixo valor comercial (Vidal et al.,

Recebido em 22 de agosto de 2016

Aceito em 3 de abril de 2017

E-mail: penelopebastos@gmail.com
2014). Assim, esforços vêm sendo direcionados para buscar fontes alternativas de dietas naturais para engorda de polvo que utilizem insumos locais e que sejam disponíveis, de baixo custo e não interfiram no mercado para consumo humano.

As maiores taxas de crescimento na engorda de O. vulgaris (18-20g.peso corporal.dia $\left.{ }^{-1}\right)$ têm sido obtidas quando são ofertados crustáceos como única dieta ou como parte de dieta mista (crustáceo Carcinus mediterraneus e peixe Bogue boops) (Aguado Giménez e García García 2002; García García e Cerezo Valverde, 2006; Prato et al., 2010).

No Brasil, apesar do enorme potencial para maricultura, a engorda de polvo é bastante incipiente e há poucas informações. Recentemente, um estudo sobre engorda experimental de $O$. vulgaris no mar, em Santa Catarina, relatou ganho em peso de 14,15 g.dia ${ }^{-1}$ com dieta mista de $40 \%$ siri Callinectes sapidus, $30 \%$ mexilhão Perna perna e $30 \%$ rejeito de pescado (Bastos et al., 2014). Contudo, a disponibilidade e o preço do siri limitam seriamente a viabilidade da engorda comercial. Em Santa Catarina, O. vulgaris é encontrado dentro das áreas de maricultura alimentando-se de bivalves cultivados. Por serem predadores naturais desses bivalves, os polvos são atraídos pela facilidade de acesso a esses moluscos como alimento ao longo do ano, principalmente de mexilhões Perna perna provenientes tanto do cultivo comercial de mexilhão quanto fixados às estruturas de apoio (balsas, bombonas, etc.) do cultivo de ostras e vieiras. A disponibilidade do $P$. perna como produto ou subproduto da 
maricultura e o valor comercial acessível tornamno um potencial item alimentar para engorda comercial do polvo como fonte de renda aos maricultores. O objetivo deste estudo foi avaliar o desempenho e o consumo da dieta de subadultos de Octopus cf. vulgaris alimentados com mexilhão Perna perna ofertado congelado e vivo, em laboratório.

Os polvos foram obtidos da engorda experimental da área aquícola do Laboratório de Moluscos Marinhos da Universidade Federal de Santa Catarina, Brasil. Nesse sistema de engorda, indivíduos subadultos de $O$. cf. vulgaris foram capturados no litoral de Florianópolis, Santa Catarina, Brasil, e mantidos por 30 dias em gaiolas flutuantes de $0,5 \mathrm{~m}^{3}$ em sistema suspenso tipo espinhel, dentro da área de cultivo de moluscos bivalves, seguindo metodologia utilizada por Bastos et al. (2014) semelhante ao aplicado no cultivo de mexilhões e densidade máxima de $5 \mathrm{~kg} \cdot \mathrm{m}^{3}$. Durante o período de manutenção nas gaiolas, os polvos foram alimentados à saciedade com dieta mista congelada de $40 \%$ siri Callinectes sp., $30 \%$ mexilhão Perna perna e $30 \%$ rejeito de pescado, ofertada uma vez ao dia.

Para o experimento, seis polvos (três machos e três fêmeas) foram transportados da área de engorda experimental no mar até o laboratório $(27,4 \mathrm{~km})$ em uma caixa de isopor de $100 \mathrm{~L}$ com aeração moderada. Dentro da caixa, os polvos foram transportados individualmente em sacos de polietileno para evitar fuga e minimizar o estresse durante o transporte. Para a manutenção dos polvos em laboratório, foi construído um sistema de recirculação semifechado composto por seis tanques circulares de $500 \mathrm{~L}$, um fragmentador de espuma, reservatório, filtro de areia e bomba hidráulica. Os polvos foram distribuídos inteiramente ao acaso, em dois grupos de três animais. Cada grupo foi mantido em um tanque circular de 500L com aeração constante, fluxo de água de $5 \mathrm{~L} \cdot \mathrm{min}^{-1} \mathrm{e}$ fotoperíodo natural (12L:12E). Foram colocados três "TES" de policloreto de vinil (PVC) com abertura de $150 \mathrm{~mm}$ de diâmetro em cada tanque como refúgio para os polvos. Os animais foram aclimatados por cinco dias e alimentados à saciedade com mexilhão $P$. perna vivo proveniente de cultivo. Segundo Lima (2010), a composição centesimal do mexilhão $P$. perna coletado em área de cultivo no litoral de Santa
Catarina é $76 \%$ de umidade, $14 \%$ de proteínas, $6 \%$ de carboidratos (Fração Nifext), 2\% de lipídios e $2 \%$ de cinzas.

Antes de iniciar o experimento, foi realizada pesagem e sexagem dos animais. $\mathrm{O}$ peso inicial (g) foi de 415,00 $\pm 123,73$ (média \pm desviopadrão). Os polvos foram mantidos nos mesmos tanques para o experimento (Fig. 1). Foram testadas duas dietas ofertadas à saciedade: grupo MC alimentado com mexilhão congelado e grupo MV com mexilhão vivo. Com relação à sexagem, foram identificados dois machos e uma fêmea no grupo MC e duas fêmeas e um macho no grupo MV.

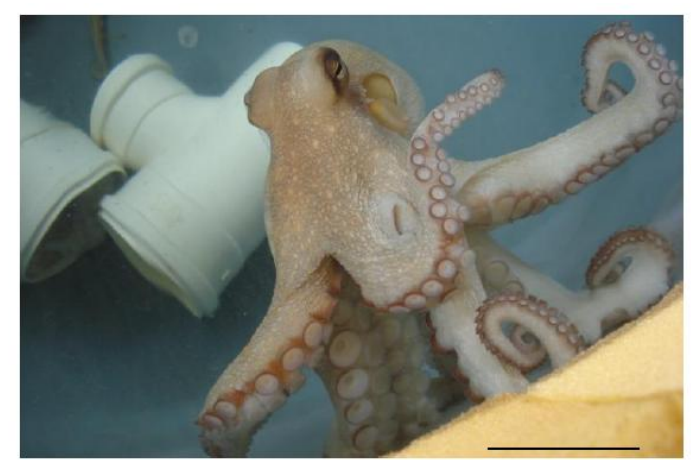

Figura 1. Polvo subadulto (Octopus vulgaris) em sistema de recirculação, em laboratório. Barra de micragem representa $10 \mathrm{~cm}$.

Os mexilhões foram selecionados por tamanho (5-7 $\mathrm{cm}$ de comprimento), limpos para retirada de fauna acompanhante e incrustações na concha e ofertados inteiros com concha, uma vez ao dia (11:00h). Os mexilhões foram distribuídos de maneira uniforme por toda a borda e área central do tanque para evitar brigas e competição dos polvos por alimento. Para a dieta MC, a porção de mexilhão foi previamente descongelada $\mathrm{e}$ mantida sob refrigeração a $6^{\circ} \mathrm{C}$. Antes de cada alimentação dos polvos, foram retirados os restos alimentares e contado o número de mexilhões consumidos. Os mexilhões encontrados abertos, cortados ou despedaçados, porém não consumidos, foram descartados. A limpeza diária do sistema foi realizada por meio de sifonagem do fundo dos tanques, renovando-se $20 \%$ do volume total do sistema. O experimento teve duração de 20 dias e a taxa de sobrevivência obtida foi de $100 \%$. 
A temperatura e a salinidade da água, verificadas diariamente, apresentaram valores (média \pm desvio-padrão) de $27,1 \pm 0,4^{\circ} \mathrm{C}$ e $30,1 \pm 0,3$ ups, respectivamente. $\mathrm{O} \mathrm{pH}$ variou entre 7,6 e 8,2 e o oxigênio foi mantido sempre acima de $90 \%$ de saturação. Os valores de temperatura e a salinidade estão dentro dos limites de tolerância da espécie (Boletzky e Hanlon, 1983). Embora a temperatura recomendada para o crescimento ótimo na engorda de $O$. vulgaris seja entre 16 e $22^{\circ} \mathrm{C}$ no Mediterrâneo (AguadoGimenez e Garcia-Garcia, 2002), por ser uma espécie cosmopolita, os limites de tolerância térmica para $O$. vulgaris podem variar conforme a localização geográfica natural ou uma dada população (Vidal et al., 2014).

Ao final do experimento, os animais foram pesados e calculou-se os índices zootécnicos: ganho em peso $(\mathrm{GP})=\mathrm{Pf}-\mathrm{Pi}$; taxa de crescimento absoluto TCA $\left(\right.$ g.dia $\left.^{-1}\right)=\mathrm{Pf} \quad \mathrm{Pi} / \mathrm{t}$; taxa de crescimento específico TCE (\% peso corporal.dia $\left.{ }^{-1}\right)=(\mathrm{LnPf}-\mathrm{LnPi}) / 100, \quad$ em que $\mathrm{Pi}=$ peso inicial $(\mathrm{g}), \mathrm{Pf}=$ peso final $(\mathrm{g}), \mathrm{t}=\mathrm{tempo}$ (dias). Para avaliar estatisticamente o efeito das dietas no desempenho entre grupos e a diferença no consumo das dietas, após ter sido verificada a homocedasticidade dos dados com teste de Levene e a distribuição normal dos dados com teste de Shapiro-Wilk, foi aplicada ANOVA unifatorial. Considerou-se o nível de significância com valores de $\mathrm{P}<0,05$.

Com relação ao desempenho, não houve diferença no ganho em peso entre os grupos $(\mathrm{P}=0,3046)$ (Tab. 1), assim como para TCA $(\mathrm{P}=0,3046)$ e TCE $(\mathrm{P}=0,5858)$.

Tabela 1. Média \pm desvio-padrão dos índices zootécnicos. Pi $(\mathrm{g})=415,00 \pm 123,73$

\begin{tabular}{cccc}
\hline & MC & MV & P \\
\hline Pf $(\mathrm{g})$ & $613,33 \pm 145,72^{\mathrm{a}}$ & $830 \pm 156,20^{\mathrm{a}}$ & 0,1538 \\
GP $(\mathrm{g})$ & $273,33 \pm 94,52^{\mathrm{a}}$ & $340 \pm 26,46^{\mathrm{a}}$ & 0,3046 \\
TCA $\left(\mathrm{g} \cdot \mathrm{dia}^{-1}\right)$ & $13,67 \pm 4,73^{\mathrm{a}}$ & $17 \pm 1,32^{\mathrm{a}}$ & 0,3046 \\
TCE $\left(\% \cdot\right.$ dia $\left.^{-1}\right)$ & $2,95 \pm 0,58^{\mathrm{a}}$ & $2,64 \pm 0,37^{\mathrm{a}}$ & 0,5858 \\
Consumo mexilhão $_{\text {Consumo mexilhão.polvo.dia }}{ }^{-1}$ & $129 \pm 31^{\mathrm{a}}$ & $100 \pm 19^{\mathrm{a}}$ & 0,2313 \\
\hline
\end{tabular}

Valores na mesma linha e com diferentes expoentes são significativamente diferentes $(\mathrm{P}<0,05)$. MC: mexilhão congelado, $\mathrm{MV}$ : mexilhão vivo, $\mathrm{Pi}=$ peso inicial, $\mathrm{Pf}=$ peso final, $\mathrm{GP}=$ ganho em peso, $\mathrm{TCA}=$ taxa de crescimento absoluto, TCE $=$ taxa de crescimento específico.

As taxas de crescimento obtidas no presente estudo são superiores aos de Biandolino et al. (2010), que obtiveram taxas de crescimento de $7,17 \pm 4,85 \mathrm{~g} \cdot \mathrm{dia}^{-1}$ e $1,19 \pm 0,95 \% \cdot$ dia $^{-1}$ com monodieta de mexilhão Mytilus galloprovincialis. Quando comparada a outras dietas naturais para O. vulgaris (Tab. 2), a TCE obtida no presente relato foi superior às alcançadas com monodieta de crustáceo Carcinus mediterraneus $\left(2,04 \% \cdot\right.$ dia $\left.^{-1}\right)$ e dieta mista de crustáceo $C$. mediterraneus e peixe Bogue boops $\left(1,98 \% \cdot \mathrm{dia}^{-1}\right)$ na engorda de subadultos de $O$. vulgaris, na Espanha (GarciaGarcia e Cerezo-Valverde, 2006). Os resultados do presente estudo também foram superiores aos de Bastos et al. (2014), que obtiveram taxas de crescimento 14,1 g.dia ${ }^{-1}$ e $1,29 \% \cdot$ dia $^{-1}$ com dieta mista de crustáceo $C$. sapidus, mexilhão $P$. perna e rejeito de pescado, em engorda experimental no mar. Entretanto, as maiores taxas de crescimento (20g.dia $\left.{ }^{-1} ; 2,66 \% \cdot \operatorname{dia}^{-1}\right)$ na engorda de $O$. vulgaris foram obtidas com monodieta de crustáceo Maja crispata (Prato et al., 2010). Essas diferenças podem estar relacionadas principalmente à composição da dieta, ao sistema de cultivo, à temperatura da água e à idade dos polvos, pois todos os indivíduos de $O$. vulgaris utilizados em engorda experimental são provenientes da captura no ambiente. Ao se compararem as taxas de crescimento obtidas no presente estudo com as relatadas para outras espécies de polvo consideradas emergentes para aquicultura (Tab. 2), os resultados do presente estudo demonstram que a TCA e a TCE para ambas as dietas (mexilhão vivo e congelado) também foram bastante superiores às relatadas para Octopus mimus (Gould, 1852) no Chile. Possivelmente, essas diferenças são devido às altas concentrações de lipídios na composição nutricional do molusco $P$. thaca e ao 
desconhecimento da temperatura ótima para $O$. mimus. Além disso, a TCE obtida no presente trabalho foi similar aos maiores valores de TCE obtidas tanto com monodieta de crustáceo quanto com dieta mista na engorda de Octopus maya (Voss e Solis Ramirez, 1966), espécie endêmica do México (Tab. 2).

Tabela 2. Efeito de diferentes tipos de dieta na engorda de Octopus sp.

\begin{tabular}{|c|c|c|c|c|c|c|}
\hline Espécie & $\begin{array}{l}\mathrm{Pi} \\
(\mathrm{g})\end{array}$ & Temperatura $\left({ }^{\circ} \mathrm{C}\right)$ & Dieta & $\begin{array}{l}\text { TCA } \\
\left(\text { g.dia }^{-1}\right)\end{array}$ & $\begin{array}{c}\text { TCE } \\
\left(\% \cdot \text { dia }^{-1}\right)\end{array}$ & Referência \\
\hline O. vulgaris & $415 \pm 124$ & $27,1 \pm 0,4$ & $\begin{array}{l}\text { mexilhão vivo } \\
\text { (Perna perna) }\end{array}$ & 17 & 2,64 & $\begin{array}{l}\text { Presente } \\
\text { estudo }\end{array}$ \\
\hline O. vulgaris & $415 \pm 124$ & $27,1 \pm 0,4$ & $\begin{array}{l}\text { mexilhão congelado } \\
\text { (Perna perna) }\end{array}$ & 13,67 & 2,95 & $\begin{array}{l}\text { Presente } \\
\text { estudo }\end{array}$ \\
\hline O. vulgaris & $499 \pm 75$ & $18,0 \pm 2,0$ & $\begin{array}{c}\text { mexilhão } \\
\text { (Mytilus } \\
\text { galloprovincialis) }\end{array}$ & 7,17 & 1,19 & $\begin{array}{l}\text { Biandolino et } \\
\text { al. (2010) }\end{array}$ \\
\hline O. vulgaris & $614 \pm 90$ & $18,0 \pm 2,0$ & $\begin{array}{l}\text { mexilhão vivo } \\
\text { (Mytilus } \\
\text { galloprovincialis) }\end{array}$ & 7,57 & 0,93 & $\begin{array}{l}\text { Prato et al. } \\
\quad(2010)\end{array}$ \\
\hline O. vulgaris & $614 \pm 90$ & $18,0 \pm 2,0$ & $\begin{array}{l}\text { crustáceo vivo (Maja } \\
\text { crispata) }\end{array}$ & 20 & 2,66 & $\begin{array}{l}\text { Prato et al. } \\
\quad(2010)\end{array}$ \\
\hline O. vulgaris & $491 \pm 26$ & $16,5 \pm 1,0$ & $\begin{array}{c}\text { crustáceo congelado } \\
\text { (Carcinus mediterraneus) }\end{array}$ & 18,83 & 2,04 & $\begin{array}{c}\text { Garcia-Garcia } \\
\text { e Cerezo- } \\
\text { Valverde } \\
(2006)\end{array}$ \\
\hline O. vulgaris & $526 \pm 133$ & $16,5 \pm 1,0$ & $\begin{array}{l}\text { dieta mista congelada } \\
\text { (crustáceo } C \text {. } \\
\text { mediterraneus e peixe } \\
\text { Bogue boops) }\end{array}$ & 18,47 & 1,98 & $\begin{array}{c}\text { Garcia-Garcia } \\
\text { e Cerezo- } \\
\text { Valverde, } \\
\text { (2006) }\end{array}$ \\
\hline O. vulgaris & $787 \pm 150$ & $25 \pm 1,7$ & $\begin{array}{l}\text { dieta mista congelada } \\
\text { (crustáceo } C \text {. sapidus, } \\
\text { mexilhão } P \text {. perna e } \\
\text { rejeito de pescado) }\end{array}$ & 14,15 & 1,29 & $\begin{array}{l}\text { Bastos et al. } \\
\quad(2014)\end{array}$ \\
\hline O. maya & 472 & 28 & crustáceo (C. sapidus) & 24 & 2,7 & $\begin{array}{l}\text { Domingues et } \\
\text { al. (2007) }\end{array}$ \\
\hline O. maya & 542 & 28 & $\begin{array}{l}\text { crustáceo (C. sapidus) e } \\
\text { peixe (Lutjanus } \text { spp.) }\end{array}$ & 17,8 & 2,4 & $\begin{array}{c}\text { Domingues et } \\
\text { al. (2012) }\end{array}$ \\
\hline O. mimus & $800-1000$ & $17-20$ & $\begin{array}{c}\text { molusco de areia fresco } \\
\text { (Protothaca thaca) }\end{array}$ & 6,6 & 0,7 & $\begin{array}{c}\text { Zuñiga et al. } \\
\text { (2011) }\end{array}$ \\
\hline
\end{tabular}

$\mathrm{Pi}=$ peso inicial, TCA=taxa de crescimento absoluto, TCE=taxa de crescimento específico.

Outro fator a ser considerado é o pequeno número de animais do presente estudo, que se deve à grande dificuldade de captura de polvos pela escassez de informações disponíveis sobre épocas e locais de ocorrência de polvos em áreas rasas e intermediárias do litoral de Santa Catarina.

Com relação ao consumo da dieta, os polvos aceitaram bem tanto mexilhão vivo quanto congelado. Observou-se, em ambos os tratamentos, que, logo após a oferta do alimento, um mesmo polvo capturou de dois a três mexilhões ao mesmo tempo, envolvendo-os com os braços e, em geral, levando-os para dentro dos refúgios. Ocasionalmente, os polvos consumiram os mexilhões no mesmo ponto do tanque em que foram ofertados ou ao redor dos refúgios. A maior parte dos mexilhões foi consumida nas primeiras três a quatro horas após serem ofertados. Observou-se também que os mexilhões congelados não consumidos nesse período, ao serem manipulados pelos polvos, despedaçaram-se com facilidade. Esses pedaços ou pequenos destroços da carne dos mexilhões também não foram consumidos posteriormente pelos polvos, mesmo estando disponíveis até que fosse realizada a limpeza do tanque, o que ocasionou maior sujidade da água no tanque que recebeu mexilhões congelados. No grupo MV, verificou-se que os mexilhões vivos não consumidos, mesmo sendo manipulados pelos polvos, não foram encontrados abertos ou perfurados, permanecendo vivos e inteiros dentro das conchas, o que permitiu maior limpeza do tanque desse grupo quando comparado ao tanque do grupo alimentado com mexilhões congelados. 
O número de mexilhões congelados consumidos foi maior quando comparado ao de mexilhões vivos (Tab. 1), porém o consumo não foi diferente entre os grupos $(\mathrm{P}=0,2313)$. $\mathrm{O}$ fato de os polvos terem consumido mais mexilhão congelado em relação ao mexilhão vivo pode estar relacionado ao maior gasto energético para abrir os mexilhões vivos ou a uma possível perda da qualidade nutricional dos mexilhões após o processo de congelamento e descongelamento antes de ser ofertado como alimento aos polvos. Segundo McQuaid (1994), juvenis de $O$. vulgaris, ao predarem $P$. perna, gastam energia tanto para capturar a presa, uma vez que o mexilhão pode estar fixado pelo bisso, quanto para perfurar a concha e romper o músculo adutor do mexilhão. De fato, como esse gasto energético não é necessário para consumir mexilhão congelado, isso pode facilitar a predação e a ingestão de um número maior de mexilhões.

Em termos práticos, é possível engordar subadultos de $O$. vulgaris com monodieta de mexilhão Perna perna ofertado congelado ou vivo. Estudos sobre digestibilidade do $P$. perna na dieta e seu efeito na composição bioquímica dos polvos poderão fornecer informações importantes para se verificar se o mexilhão supre as exigências nutricionais do polvo e o que acontece quando utilizado em diferentes proporções como componente de dietas mistas para engorda comercial como fonte de renda ao maricultor.

Palavras-chave: engorda, dieta, aquicultura, cefalópode, molusco

\begin{abstract}
The octopus Octopus cf. vulgaris is a potential species to diversify aquaculture. Due to absence of balanced commercial diet, growth of the $\mathrm{O}$. cf. vulgaris is based on natural diet with local and low-cost inputs. In Brazil, studies on experimental octopus ongrowing are recent and there is little available data. We evaluated the performance, survival and food consumption of $O$. vulgaris fed on mussel Perna perna for 20 days. Six octopuses with initial weight of $415 \pm 12.73 \mathrm{~g}$ (mean \pm standard deviation) were divided into two groups ( $n=3$ octopuses/group) according to the diet: MC Group (frozen mussels) and MV Group (live mussels). The Weight Gain of octopuses was 273.33 $\pm 94.52 \mathrm{~g}$ and $340.00 \pm 26.46 \mathrm{~g}$; the Absolute Growth Rate was $13.67 \pm 4.73$ and $17.00 \pm 1.32 \mathrm{~g} . \mathrm{dia}^{-1}$ and the Specific Growth Rate of $2.95 \pm 0.58$ and $2.64 \pm 0.37 \%$.day ${ }^{-1}$ to MC and MV groups, respectively. There was no significant difference in performance between groups and the survival rate was

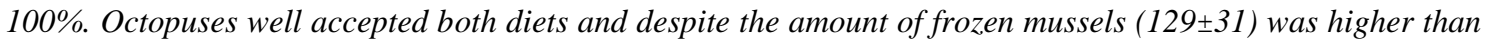
in live mussels $(100 \pm 19)$, there was no significant difference regarding the consumption between groups. Our results demonstrate that the mussel Perna perna can be used frozen or live as monodiet in $O$. cf. vulgaris ongrowing.
\end{abstract}

Keywords: ongrowing, diet, aquaculture, cephalopod, mollusk

\section{AGRADECIMENTOS}

À Dra. Aimê R. M. Magalhães (UFSC), pelo apoio nas atividades, e ao Tiê Ferreira, por ceder gentilmente os polvos. Ao Laboratório de Moluscos Marinhos (UFSC), por viabilizar este trabalho, e ao Conselho Nacional de Desenvolvimento Científico e Tecnológico e Ministério da Pesca e Aquicultura, pelo financiamento.

\section{REFERÊNCIAS}

AGUADO-GIMENEZ, F.; GARCIA-GARCIA, B. Growth and food intake models in Octopus vulgaris Cuvier (1797): influence of body weight, temperature, sex and diet. Aquacult. Int., v.10, p.361-377, 2002.
BASTOS, P.T.; BRANDÃO, A.G.; FERREIRA, J.F. et al. Engorda do polvo Octopus vulgaris em gaiolas flutuantes de pequeno volume. Agropecu. Catarin., v.27, p.51-53, 2014.

BIANDOLINO, F.; PORTACCI, G.; PRATO, E. Influence of natural diet on growth and biochemical composition of Octopus vulgaris Cuvier, 1797. Aquacult. Int., v.18, p.1163-1175, 2010.

BOLETZKY, S.; HANLON, R.T. A review of the laboratory maintenance, rearing and culture of cephalopod molluscs. Mem. Natl. Mus. Victoria., v.44, p.147-187, 1983. 
DOMINGUES, P.; LOPEZ, N.; ROSAS, C. Preliminary trials on the use of large outdoor tanks for the ongrowing of Octopus maya juveniles. Aquacult. Res., v.43, p.26-31, 2012

DOMINGUES, P.M.; LOPEZ, N.; MUÑOZ, J.A. et al. Effects of a dry pelleted diet on growth and survival of the Yucatan octopus, Octopus maya. Aquacult. Nutr., v.13, p.273-280, 2007.

ESTEFANELL, J.; ROO, J.; GUIRAO, R. et al. Efficient utilization of dietary lipids in Octopus vulgaris (Cuvier 1797) fed fresh and agglutinated moist diets based on aquaculture by-products and low price trash species. Aquacult. Res., v.44, p.93-105, 2013.

GARCÍA-GARCÍA, B.; CEREZO-VALVERDE, J. Optimal proportions of crabs and fish in diet for common octopus (Octopus vulgaris) ongrowing. Aquaculture, v.253, p.502-511, 2006.

IGLESIAS, J.; FUENTES, L. Octopus vulgaris: Paralarval Culture. In: IGLESIAS, J.; FUENTES, L.; VILLANUEVA, R. (Eds.). Cephalopod culture. Dordrecht: Springer Science e Business Media, 2014. p.427-450.

LEE, P.G. Nutrition of cephalopods: fueling the system. Mar. Freshw. Behav. Physiol., v.25, p.35-51, 1994.
LIMA, M. Avaliação das condições de processamento de mexilhões Perna perna précozidos e resfriados. 2010. 134f. Dissertação (Mestrado em Engenharia de Alimentos) Universidade Federal de Santa Catarina, Florianópolis, SC.

MCQUAID, C.D. Feeding behaviour and selection of bivalve prey by Octopus vulgaris Cuvier. J. Exp. Mar. Biol. Ecol., v.177, p.187202, 1994.

PRATO, E.; PORTACCI, G.; BIANDOLINO, F. Effect of diet on growth performance, feed efficiency and nutritional composition of Octopus vulgaris. Aquaculture, v.309, p.203211, 2010.

VAZ-PIRES, P.; SEIXAS, P.; BARBOSA, A. Aquaculture potential of the common octopus (Octopus vulgaris Cuvier, 1797): a review. Aquaculture, v.238, p.221-238, 2004.

VIDAL, E.; VILLANUEVA, R.; ANDRADE, J.P. et al. Cephalopod culture: current status of main biological models and research priorities. In: VIDAL, E.A.G. (Ed.). Advances in marine biology. Oxford: Academic Press, 2014. p.1-98.

ZÚÑIGA, O.; OLIVARES-PAZ, A.; TORRES, I. Evaluación del crecimiento del pulpo común Octopus mimus del norte de Chile alimentados con dietas formuladas. Lat. Am. J. Aquat. Res., v.39, p.584-592, 2011. 